\title{
An Investigation into Patient Non-Attendance and Use of a Short-Message Reminder System at a University Dental Clinic
}

\author{
Mark J. Storrs, BDSc, MPH; Helen M. Ramov, BOHDSc, Grad Dip Dent; \\ Ratilal Lalloo, PhD
}

Abstract: Non-attended appointments in health care facilities create inefficiencies and loss of clinical productivity: clinical teaching hours are reduced, impacting students' ability to meet the competencies necessary for professional registration. The aim of this study was to assess demographic and time-related factors for patient non-attendance at a dental school clinic in Australia. Appointment data were extracted from the patient management system for the years 2011 and 2012. Data included the status of appointment (attended, cancelled, or failed to attend [FTA]) and an array of demographic and time-related factors. Multinomial logistic regression was conducted to assess relationships between these factors and appointment status. Attendance rates were also compared by year following implementation of a short message service (SMS) reminder at the beginning of 2012. The results showed that, of 58,622 appointments booked with students during 2011 and 2012, 68\% of patients attended, 23\% cancelled, and $9 \%$ were FTA. The percentage of non-attended (cancelled or FTA) appointments differed by demographic and time-related factors. Females were 7\% less likely to be FTA, those aged 16-24 years were five times more likely to be FTA, and early morning appointments were $18 \%$ less likely to be cancelled and FTA. With the SMS reminder system, the odds of a cancellation were $15 \%$ higher, but FTAs were 14\% lower (both were statistically significant differences). This study found that failing to attend an appointment was significantly related to a number of factors. Clinical scheduling and reminder systems may need to take these factors into account to decrease the number of teaching hours lost due to patients' missing their appointments.

Dr. Storrs is an associate member of the Population and Social Health Research Program, Menzies Health Institute of Queensland and Lecturer in General Dental Practice, School of Dentistry and Oral Health, Griffith University, Queensland, Australia; Dr. Ramov is a general dentist in private practice in Victoria, Australia; and Dr. Lalloo is Associate Professor, School of Dentistry, University of Queensland, Queensland, Australia and Adjunct Associate Professor, Australian Research Centre for Population Oral Health, University of Adelaide, South Australia, Australia. Direct correspondence to Dr. Mark Storrs, School of Dentistry and Oral Health, Griffith Health Centre, Gold Coast Campus, Griffith University, Queensland, 4222, Australia; +61 (0)7 5678 0755; m.storrs@griffith.edu.au.

Keywords: dental education, dental school clinics, clinical education, clinic management, patients, management system, dental appointments

Submitted for publication 3/8/15; accepted 6/10/15

I n order to obtain mandatory practitioner registration with the Australian Health Practitioner Regulation Agency (AHPRA), oral health students in Australia must demonstrate they have reached a high level of professional competence and conduct and possess a variety of required attributes at the completion of their studies..$^{1,2}$ These attributes are professionalism, communication and social skills, critical thinking, health promotion abilities, scientific and clinical knowledge, and patient care skills including clinical information-gathering, diagnosis, management planning, clinical treatment, and evaluation., To master these attributes and satisfy the necessary competencies prior to graduation, students must have a sufficient number of clinical contact hours with patients in the university clinics.
Non-attended appointments can not only challenge students' ability to meet these requirements but can create significant problems for health care facilities in the resulting loss in clinical productivity and inefficiencies such as increased waiting times to secure an appointment. The majority of studies on non-attendance have focused on general practice and specialist health clinics, with fewer studies conducted on patient non-attendance at dental facilities. ${ }^{5-11}$ Two studies in the United Kingdom estimated the medical general practice non-attendance rate to be $6.5 \%{ }^{12}$ and $7.7 \% .{ }^{13}$ Non-attendance rates for primary health care in the United States range from 5\% to 55\%. ${ }^{14-19}$

Similarly, the prevalence of non-attended dental appointments has been reported as varying from $5 \%$ to $50 \% .^{9,20-29}$ A study investigating dental 
attendance patterns in a teaching hospital reported that $43.8 \%$ of patients did not attend a recall appointment. ${ }^{24}$ Awartani reported that patients who did not attend scheduled appointments with oral health students diminished the clinical teaching hours available for students to meet their goals and the requirements for registration..$^{22}$ Factors that have been found to inhibit appointment attendance include forgetting appointments, ${ }^{11}$ cost, ${ }^{24}$ lack of time, ${ }^{24,30}$ waiting time to book an appointment,,$^{31,32}$ anxiety or dental phobias, ${ }^{30,33}$ lack of transportation, ${ }^{6,34,35}$ weather, and travel distance. ${ }^{24,35}$ Demographic factors associated with non-attendance of appointments at a rural student dental clinic training facility included gender, age, patient status (private or public), and day/time of appointment. ${ }^{36}$ A suggested method to improve attendance rates has been the use of an appointment reminder service sent through postal mail, a short message service (SMS), or telephone calls. ${ }^{5,23,37,38}$ Health care practices using these services have reported improved attendance rates for both medical and dental appointments.

Paige and Mansell found that non-attendance was related to multiple barriers in seeking treatment, which in combination can increase the likelihood of failure to attend. ${ }^{32}$ However, a major limitation of most studies assessing factors associated with nonattendance is that data have been collected through self-report - the accuracy of which depends upon patient recall. In addition, the majority of studies that have assessed the use of SMS reminders have been conducted in medical clinics, with few being conducted in either dental clinics or dental educational facilities.

An understanding of the nature of non-attendance is likely to assist in maximizing use of the number of clinical dental teaching hours available and improve the efficiency of clinical dental education. By using routinely collected data, the aim of this study was to investigate the demographic and time-related risk factors for non-attended appointments at a university dental clinic. Non-attended appointments include those cancelled and those the patient failed to attend (FTA). In early 2012, the clinic implemented an SMS reminder system that sent reminder messages to patients' cell phones 24 hours before their appointment. For those patients who did not have a cell phone, the message was sent to a friend or family member designated by the patient. Available resources prohibited reminders being sent by email or a telephone call. As the reminder service commenced midway through the time our retrospec- tive data were collected, there was an opportunity to assess the effectiveness of the SMS reminder service in reducing non-attended dental appointments as a secondary aim.

\section{Materials and Methods}

Ethical approval to access, analyze, and report patient information from 2011 and 2012 in a de-identified format was granted by the Griffith University Human Research Ethics Committee (GU Ref No: DOH/35/12/HREC). The Griffith University School of Dentistry and Oral Health (DOH) clinic was established in 2004 on the Gold Coast, Australia. ${ }^{39}$ During the years 2011 and 2012, the university clinic had 54 chairs and operated five days per week from January through November.

In response to a number of educational challenges, the school introduced an intraprofessional curriculum for undergraduate dentistry, oral health therapy, and dental technology students. Under this model, students from each oral health profession collaborate as a team when planning and managing patient treatment in both the clinic and laboratory. ${ }^{40}$ In the undergraduate dental clinic, preventive services are supplied by third-year oral health therapy students, and general dental services are provided by third-, fourth-, and fifth-year dental students.

Patients attending the clinic are first screened by a registered dentist to assess their suitability to be managed by a team of students. If a course of care can be adequately managed by a team of students, an appointment is booked with an appropriate student for a comprehensive dental examination. Subsequent to the dental examination and upon receiving informed consent to conduct a particular treatment plan, a number of other appointments are scheduled to facilitate timely completion of the plan. In 2011 and 2012, at least 25,000 appointments with students were scheduled per annum at DOH.

The sample analyzed in this study consisted of all patients who booked a dental appointment with students at the undergraduate university clinic in 2011 and 2012. Retrospective data were obtained from the DOH patient management system, Titanium by Spark Dental Technology, which stores patient records and information associated with appointment times. The system showed that 27,203 and 33,497 appointments (total 60,700) were potentially available for, respectively, 229 undergraduate students treating patients in 2011 and 247 students in 2012. 
The system administrator extracted the following data for each appointment booked over the two-year study period: patient age and gender, payor type (public or private), residential location based on postcode, appointment type (emergency, endodontics, examination, operative, oral surgery, periodontal, preventive, prosthetic), and appointment time (month, day of week, and time of day). Patients' age was categorized into the following groups: 16$24,25-34,35-44,45-54,55-64$, and $65+$ years. All postcodes were recoded into two categories: patients residing on the Gold Coast and those outside of the Gold Coast. The time of cancellations was grouped into those made less than or equal to 24 hours and those made greater than 24 hours before a scheduled appointment.

All data for this time period were downloaded into the Statistical Package for the Social Sciences (SPSS) version 21.0 (IBM Corp., Armonk, NY, USA) for verification, cleaning, and statistical analysis. The proportion of missing data was evaluated to assess the effect on the findings. From the potential 60,700 appointments available in 2011 and 2012, 58,622 (96.6\%) appointments were booked. An analysis of missing data indicated that the variable "type of appointment booked" contained 7,571 (12.9\%) missing values from the bookings made in both years. All other variables had missing data that comprised less than $1.4 \%$ of the overall data. A frequency distribution was calculated for each of the patient characteristics, appointment time and type, and both non-attended (FTA and cancelled) and attended appointments. Bivariate analyses were conducted to determine the significance of difference among cancelled, FTA, and attended appointments for each of the patient demographic and appointment time characteristics. To assess the relationship between non-attendance and the other characteristics, a multinomial logistic regression model was used with all of the variables entered into an adjusted model. Both unadjusted (not presented) and adjusted odds ratios were calculated to measure associations. Odds ratios with a $95 \%$ confidence interval, excluding the value 1 , were considered statistically significant.

A logistic regression assessed the effects of the SMS reminder service upon appointment status and time of cancellation. The resultant chi-square test of contingencies showed if there was a significant difference for attendance rates and time of cancellation between the 2011 data and that following introduction of the SMS reminder service in 2012.

\section{Results}

In the two-year study period, 58,622 appointments in total were booked at $\mathrm{DOH}$ for 8,864 patients. A total of $39,917(68.1 \%)$ appointments were attended, 13,366 (22.8\%) were cancelled, and $5,339(9.1 \%)$ were FTA (Table 1). Of the booked appointments, $56 \%$ were for females, $54.8 \%$ were for persons aged 45 years and over, $71.6 \%$ were for patients with public funding, $90.8 \%$ were for Gold Coast residents, and $47.1 \%$ were to receive operative care and $11.4 \%$ examinations.

The age of the patient was a significant factor related to cancelled and especially FTA appointments. Compared to an FTA rate of $4.2 \%$ for patients 65 years of age and over, for young adults (16-24 years) the FTA rate was about $15 \%$ (Table 1). Patients aged 16-24 years were almost five times more likely (95\% CI 4.22-5.52) to be FTA compared to the oldest group of patients. Females were 1.16 times more likely (95\% CI 1.11-1.21) to cancel and 7\% less likely (95\% CI 0.87-0.99) to be FTA compared to males. Public patients were $16 \%$ less likely to cancel $(95 \%$ CI 0.80-0.88) than patients paying privately, and patients residing outside the Gold Coast were 19\% less likely to be FTA (95\% CI 0.71-0.91) compared to those living within the Gold Coast.

Appointments booked as an emergency had the highest percentage of attendance (77.5\%), and those booked as an endodontic appointment had the lowest proportion of attendance $(61.0 \%)$ and the highest proportion of FTA $(10.2 \%)$ (Table 1$)$. The odds of a cancellation and FTA were 0.34 (95\% CI 0.30-0.39) and 0.67 (95\% CI 0.55-0.81) lower, respectively, for emergency appointments compared to prosthodontic appointments. The appointment category with the highest odds of a cancellation was periodontal appointments, which had an odds ratio of $1.17(95 \% \mathrm{CI}$ 1.04-1.31) compared to prosthodontic appointments.

The month of June had the highest FTA percentage (11.7\%), and appointments booked in February, the first month of the academic year, had the lowest $(8.0 \%)$. The FTA proportion was highest for appointments booked on a Monday (10.2\%) and lowest for those booked on a Thursday (7.7\%); however, the percentage of attended appointments was similar across the days of the week (Table 1). The odds of being FTA were less than one for all weekdays when compared to Monday, with patients being 33\% less likely to be FTA (95\% CI 0.60-0.74) on Wednesday. The last appointment of the day, scheduled between 
5:30 and 7:00 pm, had the highest proportion of FTA patients $(10.6 \%)$, whereas appointments booked in the 12 noon to $1: 30 \mathrm{pm}$ session had the lowest FTA percentage $(7.7 \%)$ but the highest cancellation percentage (24.1\%). The percentage of attended appointments was similar across the appointment sessions. Patients booked in the earliest appointment session between 8:00 and 9:30 am were 18\% less likely to both cancel $(95 \%$ CI $0.76-0.89)$ and be FTA $(95 \%$ CI 0.74-0.92) compared to patients booked in the 5:30 to 7:00 pm session. Those booked in the 12 noon to $1: 30 \mathrm{pm}$ session were $25 \%$ less likely to be FTA (95\% CI 0.67-0.83) compared to patients booked in the latest appointment session.

In assessing the effectiveness of the SMS reminder system, we found the odds of a cancellation were 1.15 (95\% CI 1.10-1.20) times higher in 2012 compared to 2011 , whereas patients were $14 \%$ less likely to be FTA (95\% CI 0.81-0.92) in 2012 (Table 1). A Pearson's chi-square test of contingencies (with $\alpha=0.05$ ) was used to evaluate whether appointment status was related to introduction of the SMS reminder system. A lower proportion of FTA appointments $(2,532 / 8.4 \%$ versus $2,807 / 9.9 \%)$ and a higher proportion of cancellations $(7,202 / 23.8 \%$ versus $6,164 / 21.7 \%$ ) occurred after introducing the SMS reminder system in 2012 compared to 2011. However, attendance at appointments was slightly higher in 2011 (68.4\%) than $2012(67.8 \%)$. The chi-square test was statistically significant, $\chi^{2}(2, \mathrm{~N}=58,622)=$ $66.1, p<0.001$. Patients were more likely to cancel appointments and less likely to be FTA after introduction of the reminder system (Figure 1).

The Pearson chi-square test of contingencies also assessed whether the cancellation time prior to a scheduled appointment was related to presence of the SMS reminder system. Table 2 shows the proportion of cancellations made less than or equal to 24 hours and those made greater than 24 hours before a scheduled appointment for 2011 and 2012. The findings indicate that the proportion of cancellations made less than or equal to 24 hours before a scheduled appointment increased in $2012(72.2 \%)$ compared to $2011(63.3 \%)$. The chi-square test was statistically significant, $\chi^{2}(1, N=13,366)=120.6, p<0.001$.

\section{Discussion}

Empirical data analyzed in this study were collected primarily by receptionists and entered into Titanium upon contact with patients who were mak- ing or cancelling bookings. At the time of booking, undergraduate oral health students and their clinical supervisors also recorded demographic information, appointment status, and most time-related indicators, except for cancellation times, through Titanium. Data collected were deemed valid and reliable as none were based on self-report and the amount of data missing was small (1.4\%) except for the indicator "type of appointment booked." The higher amount of data missing for that item may have been due to students' and supervisors' omitting to record the type of procedure intended to be performed when patients did not attend a booked appointment. The effect of missing data on both the magnitude and significance of associations in this study was considered negligible due to the large sample size included.

The age of the patient was found to be the most important factor related to non-attended appointments in this study. Younger (16 to 34 years) patients were almost five times more likely to be FTA compared to the eldest patient group. This statistically significant difference is consistent with the majority of studies that have also found younger patients to be more likely to not attend appointments. . $^{1,24,36,38,41-43}$ The strong evidence of non-attendance among younger patients suggests that specific strategies to improve patient attendance for this group need to be implemented. Improvement may require more than an SMS reminder, such as a telephone call more than 24 hours in advance of the appointment followed up with the SMS reminder 24 hours in advance of the appointment. If non-attendance in the younger group were reduced, the number of clinical teaching hours lost would be considerably reduced.

Other factors related to non-attendance and amenable to intervention were the type of appointment, day of week, and time of day. Cancellations were particularly high for endodontic and periodontic appointments, so the importance of attending those appointments needs to be emphasized with patients when discussing their treatment plan. For both types of appointments, but especially in the case of endodontics, the higher rates of non-attendance could be due to higher levels of dental fear and anxiety related to endodontic treatment. ${ }^{19}$ It may also be due to the resolution of symptoms that patients experience after the first endodontic appointment as some patients may be less inclined to attend a further appointment if they believe treatment has been completed. This possibility is supported by the study conducted by Paige and Mansell, which found that patients were less likely to attend future appointments if they 


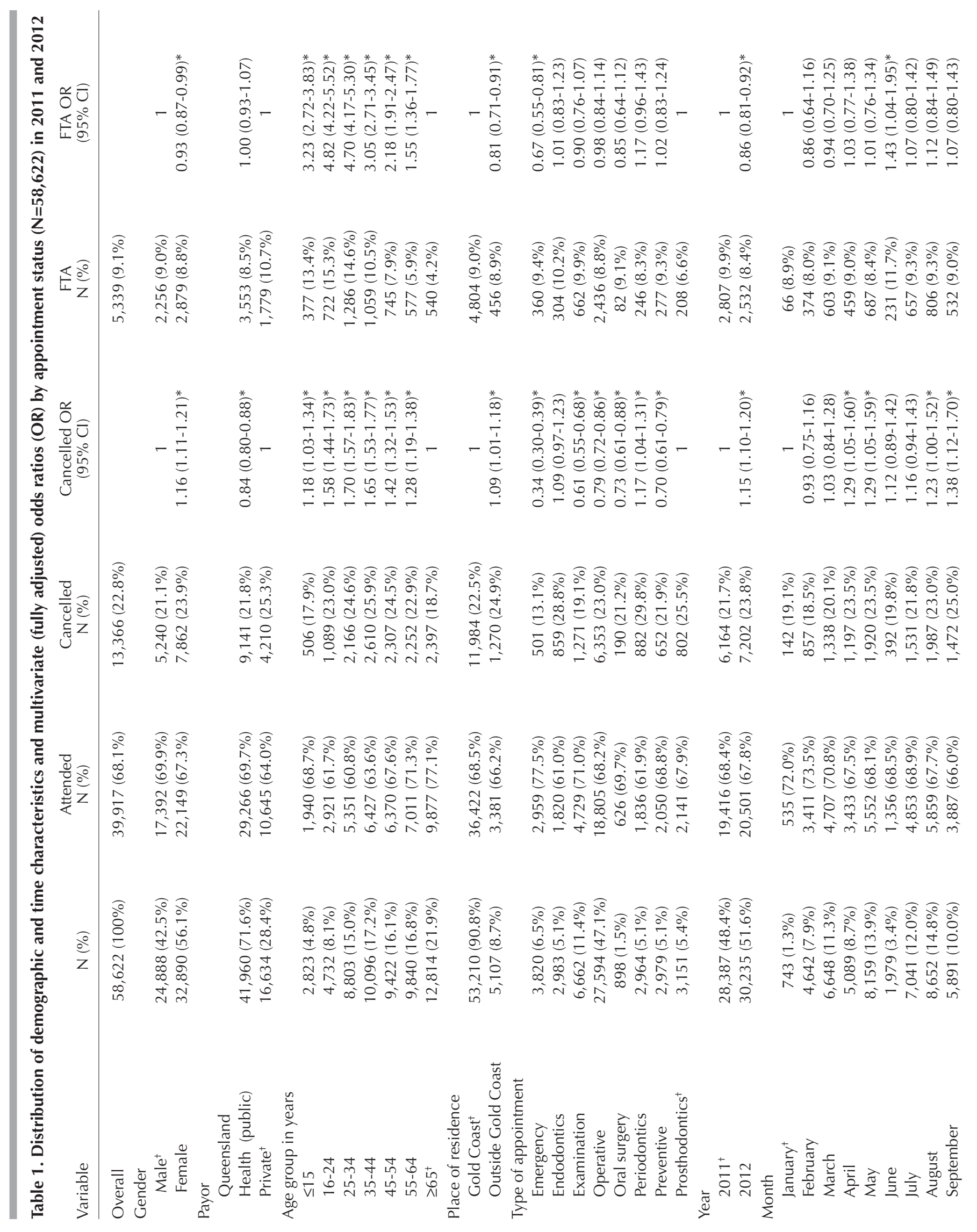



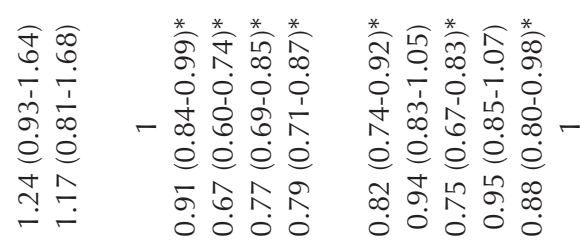

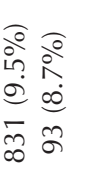
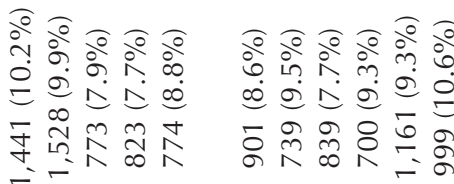

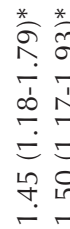
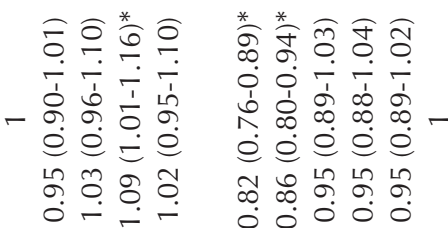

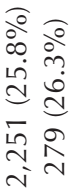
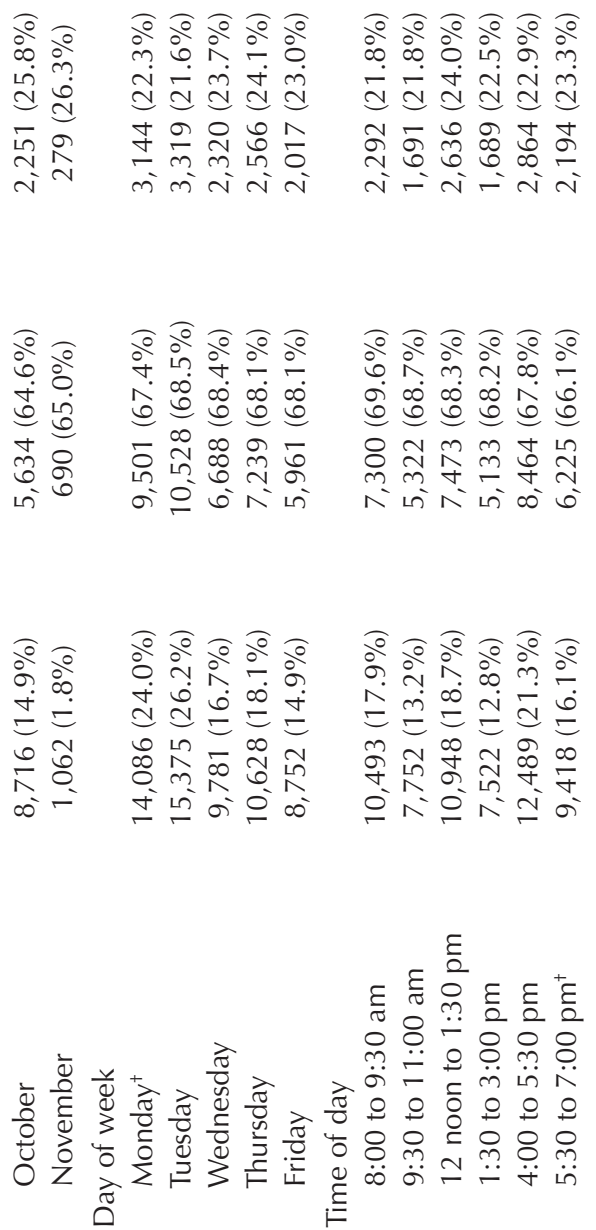

experienced an improvement in the symptoms of a condition in which ongoing care was required. ${ }^{32}$

Some significant associations were found in our study between particular appointment booking times and non-attendance indicators, in contrast to other studies that reported no significant differences in attendance rates. ${ }^{36,38,44}$ Our study's findings showed that appointments booked in the 5:30 to 7:00 pm time frame had the largest proportion of FTA appointments. This higher number could be due to family commitments, transportation difficulties, and/ or tiredness at that time. Appointments booked for the 8:00 to $11: 00$ am time frame also had significantly lower odds of a cancellation in comparison to those booked for 5:30 to 7:00 pm. The one study that found a significant association between appointment time and non-attendance reported a decrease in cancellations as the morning progressed, ${ }^{45}$ which is opposite to our findings.

Another appointment characteristic found to be associated with non-attendance in our study was the day of the week the appointment was booked. Monday had the largest number of FTA appointments, which is consistent with the findings of Vikander et al. ${ }^{17}$ In comparison to Monday, all other days of the week had significantly lower odds of an FTA occurring, with Tuesday having the lowest odds $(\mathrm{OR}=0.67, \mathrm{CI} 0.60-0.74)$. As our findings suggest that non-attendance is most likely to occur on a Monday and at the latest appointment in the day, adjustments to the booking system can be made to minimize the number of non-attended appointments. As the DOH postgraduate clinics are underutilized before 4:00 $\mathrm{pm}$, consideration may be given to booking fewer appointments in the latest appointment of the day and additional bookings at earlier times. A number of student sessions on a Monday evening could be redeployed to an alternative session and/or another evening session through utilizing maximum clinical space when the proportion of non-attended appointments is low.

A greater percentage of patients who paid privately for their treatment failed to attend compared to public patients whose fees were paid by the state health department (Queensland Health). However, this association was not significant. In Australia, patients seeing students for dental treatment either pay privately for their treatment from their own funds or, as public patients, the state covers fees for most procedures. Patients who pay privately may have dental health insurance; however, insurance companies in Australia will not cover treatment performed 


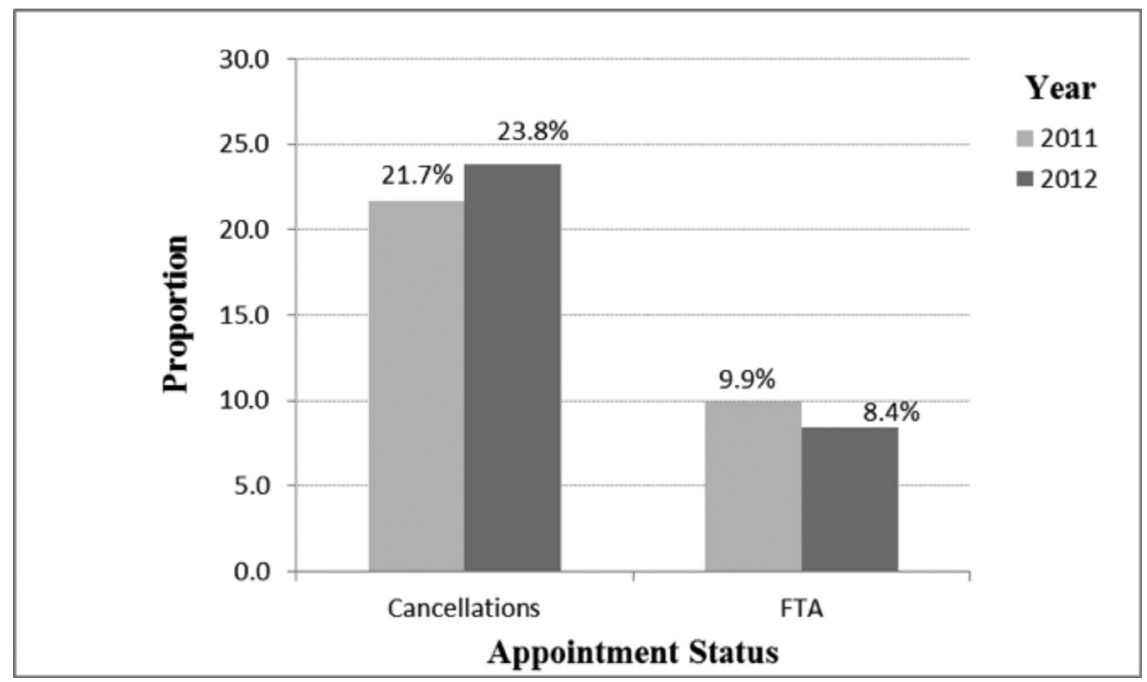

Figure 1. Comparison of appointment status before (2011) and after introduction (2012) of reminder service

by students under supervision. Public patients are those on social welfare support, whose eligibility to receive state support is deemed by a government income means test. These patients include pensioners, students, and those earning low incomes; thus, they may have fewer work commitments and/or work-related emergencies that impact their time and therefore may be less inclined to be FTA. Public patients who are FTA consistently may also jeopardize receipt of their welfare support, which would serve as a deterrent to being FTA. Private patients confronted by an unexpected expense not associated with their dental treatment may deem saving funds for that expense to be a priority and therefore be

Table 2. Comparison of cancellations made less than and greater than $\mathbf{2 4}$ hours before an appointment scheduled in 2011 and 2012, by number and percentage of total

\begin{tabular}{lccc} 
Year & $\leq 24$ Hours & $\begin{array}{c}\text { Cancellations } \\
>24 \text { Hours }\end{array}$ & Total \\
\hline 2011 & 3,904 & 2,260 & 6,164 \\
& $(63.3 \%)$ & $(36.7 \%)$ & $(100 \%)$ \\
2012 & 5,202 & 2,000 & 7,202 \\
& $(72.2 \%)$ & $(27.8 \%)$ & $(100 \%)$ \\
Total & 9,106 & 4,260 & 13,366 \\
& $(68.1 \%)$ & $(31.9 \%)$ & $(100 \%)$
\end{tabular}

$\chi^{2}(1, N=13,366)=120.6, p<0.001$
FTA for an appointment rather than face offering an excuse for cancelling.

The use of the SMS reminder service was found to be associated with a significant decrease in the proportion of FTA appointments as the odds of being an FTA were 14\% less in 2012 than 2011. This difference is consistent with the findings of the majority of studies that suggest the use of an SMS reminder will decrease non-attendance; however, those studies were mostly conducted with hospital outpatients..$^{5,37,46,47}$ The findings of our study are consistent with two ${ }^{23,48}$ of the four studies that assessed SMS reminders in dental clinics. ${ }^{9,23,48,49}$ Similar results were found in the study conducted by Foley and O'Neill, which also used a historical group from one year earlier as a comparison. ${ }^{23}$ Their study was conducted at a university pediatric clinic and reported a reduction in non-attendance of $13.5 \%$ for the postgraduate pediatric clinic and $4.8 \%$ for the pediatric staff dentists. Although our study found a much lower reduction in FTA appointments $(1.5 \%$ decrease $)$, the difference was statistically significant. The much larger reduction in non-attendance in the Foley and O'Neill study could be due to the presence of a high nonattendance rate in the comparison groups $(23.9 \%$ for the postgraduate dental clinic and $29.2 \%$ for pediatric dentists). Those higher rates carried a greater potential for a reduction in the number of non-attended appointments when an SMS reminder was used. 
The study conducted by Perry at a National Health Service dental access center in Scotland that served patients of all ages reported a significant $16.6 \%$ reduction in FTA appointments after SMS reminders were introduced. ${ }^{27}$ Although that study also reported a higher rate of FTA reduction compared to our study, the Perry study involved only two dentists and did not indicate the time lag between the reminder service being introduced and the audit of patient attendance. We found no studies involving patients of all ages in a comparable university dental clinic, so that a reliable assessment of FTA rates after introducing an SMS reminder service could be conducted. However, irrespective of the patient's age, it is likely that SMS reminder messages would have been sent to the cell phone of an adult caregiver who would then be responsible for any consequent attendance. In this regard, comparison of FTA rates in studies involving patients of different ages may still yield important information. However, we acknowledge that particular issues pertaining to very young and old patients, such as sickness, may suddenly impact attendance, whatever the intention to attend of those receiving the reminder. Therefore, replication studies that audit dental patients of all ages in similar settings should be conducted to provide reliable comparisons.

Studies generally do not report the effect of SMS reminders on cancelled appointments. The results of our study found that the use of an SMS reminder had a significant association with an increased number of cancellations and, in particular, the proportion of cancellations made less than or equal to 24 hours before a scheduled appointment. This effect is most likely because patients who may have forgotten about their appointment were reminded and given the opportunity to cancel or reschedule. Prior to introduction of the SMS reminder service, forgotten appointments would have most likely been an FTA rather than a cancellation. It may be postulated that, by sending SMS reminders earlier, cancellations would be received sooner, allowing time for those cancelled appointments to be filled with other patients. However, further investigation utilizing a control group and groups of patients receiving SMS reminders at different time intervals would provide empirical evidence for the optimum time at which to remind patients prior to their scheduled appointment.

The generalizability of findings from this study is limited as patient attendance rates cannot be applied to practices outside of a university setting. As the findings pertain to $\mathrm{DOH}$, this study should be repeated in similar university dental clinics, both nationally and internationally, to compare findings and further suggest strategies to minimize patient non-attendance and maximize available clinical teaching hours.

\section{Conclusion}

The findings of this study were that males, younger patients (16 to 24 years of age), and those residing on the Gold Coast were most likely to fail to appear for appointments and that such failures to appear for appointments were more likely to occur in the late afternoon and on a Monday. Our evaluation of patient attendance and the use of SMS reminders revealed that these reminders were associated with a lower proportion of patients' failing to attend appointments. Clinical scheduling and reminder systems may need to be altered to take these findings into account to decrease the number of clinical teaching hours lost due to patients' non-attendance of their appointment. However, replication studies in comparable contexts, both nationally and internationally, are required before suggested strategies to reduce patient non-attendance in all university dental clinics can be generalized.

\section{Acknowledgments}

This research was supported by an undergraduate research grant from the Australian Dental Research Foundation, Inc.

\section{Disclosure}

The authors declared that they have no competing interests.

\section{REFERENCES}

1. Australian Health Practitioner Regulation Agency. Dental Board of Australia registration standards. 2014. At: www.dentalboard.gov.au/Registration-Standards.aspx. Accessed 5 June 2014.

2. Health Workforce Australia. Scope of practice review: oral health practitioners. Adelaide: Health Workforce Australia, 2011.

3. Australian Dental Council. Professional attributes and competencies of the newly qualified dentist. 2010. At: www.adc.org.au/documents/Attributes\&Competencies Dentist $\% 20 v 1.0 \% 20$ Final $\% 2010-06-11 \% 20$ Updated $\% 20$ July\%202013.pdf. Accessed 18 May 2015.

4. Australian Dental Council. Professional attributes and competencies of the newly qualified oral health therapist 2011. At: http://adc.org.au/documents/Attributes $\% 20$ $\& \% 20$ Competencies_Oral $\% 20$ Health $\% 20$ Therapist $\% 20$ v1.0\%20Final $\% 2011-06-17$.pdf?PHPSESSID $=8063 \mathrm{~d} 19$ 7fc04f4def968413fd440496b. Accessed 18 May 2015. 
5. Downer SR, Meara JG, Da Costa AC. Use of SMS text messaging to improve outpatient attendance. Med J Aus 2005; 183(7):366-8.

6. George A, Rubin G. Non-attendance in general practice: a systematic review and its implications for access to primary health care. Fam Pract 2003;20(2):178-84.

7. Haana V, Sethuraman K, Stephens L, et al. Case cancellations on the day of surgery: an investigation in an Australian paediatric hospital. ANZ J Surg 2009;79(9):636-9.

8. Kavanagh AM, Simpson JM. Predicting nonattendance for colposcopy clinic follow-up after referral for an abnormal Pap smear. Aust N Z J Public Health 1996;20(3):266-71.

9. Nelson TM, Berg JH, Bell JF, et al. Assessing the effectiveness of text messages as appointment reminders in a pediatric dental setting. J Am Dent Assoc 2011;142(4):397-405.

10. Can S, Macfarlane T, O'Brien KD. The use of postal reminders to reduce non-attendance at an orthodontic clinic: a randomized controlled trial. Br Dent J 2003;195(4): 199-201.

11. Reekie D, Devlin H. Preventing failed appointments in general dental practice: a comparison of reminder methods. Br Dent J 1998;185(9):472-4.

12. Waller J, Hodgkin P. Defaulters in general practice: who are they and what can be done about them? Fam Pract 2000;17(3):252-3.

13. Neal RD, Lawlor DA, Allgar V, et al. Missed appointments in general practice: retrospective data analysis from four practices. Br J Gen Pract 2001;51:830-2.

14. Goldman L, Freidin R, Cook EF, et al. A multivariate approach to the prediction of no-show behavior in a primary care center. Arch Intern Med 1982;142(3):563-7.

15. Gruzd D, Shear CL, Rodney WM. Determinants of noshow appointment behavior: the utility of multivariate analysis. Fam Med 1986;18(4):217-20.

16. Smith CM, Yawn BP. Factors associated with appointment keeping in a family practice residency clinic. J Fam Pract 1994;38(1):25.

17. Vikander T, Parnicky K, Demers R, et al. New patient no-shows in an urban family practice center: analysis and intervention. J Fam Pract 1986;22(3):263-8.

18. Weingarten N, Meyer DL, Schneid JA. Failed appointments in residency practices: who misses them and what providers are most affected? J Am Board Fam Pract 1997;10(6):407-11.

19. Dervin JV, Stone DL, Beck CH. The no-show patient in the model family practice unit. J Fam Pract 1978;7(6): 1177-80.

20. Albarakati SF. Appointments failure among female patients at a dental school clinic in Saudi Arabia. J Dent Educ 2009; 73(9):1118-24.

21. Almog DM, Devries JA, Borrelli JA, Kopycka-Kedzierawski DT. The reduction of broken appointment rates through an automated appointment confirmation system. J Dent Educ 2003;67(9):1016-22.

22. Awartani F. Broken appointment behavior in a dental school environment. J Contemp Dent Pract 2003;4(4): 100-7.

23. Foley J, O’Neill M. Use of mobile telephone short message service (SMS) as a reminder: the effect on patient attendance. Eur Archiv Paediatr Dent 2009;10(1):15-8.
24. George AC, Hoshing A, Joshi NV. A study of the reasons for irregular dental attendance in a private dental college in a rural setup. Indian J Dent Res 2007;18(2):78-81.

25. Horsley BP, Lindauer SJ, Shroff B, et al. Appointment keeping behavior of Medicaid vs non-Medicaid orthodontic patients. Am J Orthod Dentofacial Orthop 2007;132(1):49-53.

26. Iben P, Kanellis MJ, Warren J. Appointment-keeping behavior of Medicaid-enrolled pediatric dental patients in eastern Iowa. Pediatr Dent 2000;22(4):325-9.

27. Perry JG. A preliminary investigation into the effect of the use of the short message service (sms) on patient attendance at an NHS dental access centre in Scotland. Prim Dent Care 2011;18(4):145-9.

28. Skaret E, Raadal M, Kvale G, Berg E. Missed and cancelled appointments among 12-18-year-olds in the Norwegian public dental service. Eur J Oral Sci 1998;106(6):1006-12.

29. Wang NJ, Aspelund GO. Children who break dental appointments. Eur Arch Paediatr Dent 2009;10(1):11-4.

30. Taani DSMQ. Dental anxiety and regularity of dental attendance in younger adults. J Oral Rehabil 2002;29(6):604-8.

31. Collins J, Santamaria N, Clayton L. Why outpatients fail to attend their scheduled appointments: a prospective comparison of differences between attenders and nonattenders. Aust Health Rev 2003;26(1):52-63.

32. Paige L, Mansell W. To attend or not attend? A critical review of the factors impacting on initial appointment attendance from an approach-avoidance perspective. J Ment Health 2013;22(1):72-82.

33. Pohjola V, Lahti S, Vehkalahti MM, et al. Association between dental fear and dental attendance among adults in Finland. Acta Odontol Scand 2007;65(4):224-30.

34. Naderi S, Barnett B, Hoffman RS, et al. Factors associated with failure to follow-up at a medical clinic after an ED visit. Am J Emerg Med 2012;30(2):347-51.

35. Taani DSQ. Dental fear among a young adult Saudian population. Int Dent J 2001;51(2):62-6.

36. Lalloo R, McDonald J. Appointment attendance at a remote rural dental training facility in Australia. BMC Oral Health 2013;13(1):1-8.

37. Chen Z, Fang L, Chen L, Dai H. Comparison of an sms text messaging and phone reminder to improve attendance at a health promotion center: a randomized controlled trial. J Zhejiang Univ Sci B 2008;9(1):34-8.

38. Lehmann TNO, Aebia A, Lehmann D, et al. Missed appointments at a Swiss university outpatient clinic. Public Health 2007;121(10):790-9.

39. Evans J, Henderson AJ, Johnson NW. The future of education and training in dental technology: designing a dental curriculum that facilitates teamwork across the oral health professions. Br Dent J 2010;208(5):227-30.

40. Mattheos N, Storrs MJ, Foster LA, Oberholzer T. Intraprofessional, team-based treatment planning for oral health students in the comprehensive care clinic. J Dent Educ 2012;76(12):1589-99.

41. Hamilton W, Round A, Sharp D. Patient, hospital, and general practitioner characteristics associated with non-attendance: a cohort study. Br J Gen Pract 2002;52(477):317-9.

42. Reti S. Improving outpatient department efficiency: a randomized controlled trial comparing hospital and generalpractice telephone reminders. N Z Med J 2003;116(1175). 
43. Sheiham A, Maizels J, Cushing A, Holmes J. Dental attendance and dental status. Community Dent Oral Epidemiol 1985;13(6):304-9.

44. Hull AM, Alexander DA, Morrison F, McKinnon JS. A waste of time: non-attendance at out-patient clinics in a Scottish NHS Trust. Health Bull (Edinb) 2002;60(1):62-9.

45. Norris JB, Kumar C, Chand S, et al. An empirical investigation into factors affecting patient cancellations and no-shows at outpatient clinics. Decision Support Systems 2014;57(1):428-43.

46. Grover S, Gagnon G, Flegel KM, Hoey JR. Improving appointment-keeping by patients new to a hospital medi- cal clinic with telephone or mailed reminders. Can Med Assoc J 1983;129(10):1101-3.

47. Hasvold PE, Wootton R. Use of telephone and SMS reminders to improve attendance at hospital appointments: a systematic review. J Telemed Telecare 2011;17(7):358-64 .

48. Prasad S, Anand R. Use of mobile telephone short message service as a reminder: the effect on patient attendance. Int Dent J 2012;62(1):21-6.

49. Bos A, Hoogstraten J, Prahl-Andersen B. Failed appointments in an orthodontic clinic. Am J Orthod Dentofacial Orthop 2005;127(3):355-7. 